03

\title{
Возможность формирования двухлепестковых вихревых световых полей с помощью модифицированного ЖК фокусатора*
}

\author{
(C) С.П. Котова, А.М. Майорова, С.А. Самагин \\ Самарский фрилиал ФИАН, \\ 443011 Самара, Россия \\ e-mail: kotova@fian.smr.ru
}

Поступила в редакцию 24.09.2018 г.

Проанализированы возможности формирования световых полей с распределением интенсивности в виде двух максимумов, вращающихся в процессе распространения, с помощью жидкокристаллического модального устройства. Оцениваются скорости поворота распределения интенсивности в зависимости от радиуса кривизны падающей на модулятор волны, а также энергетическая эффективность сформированных полей.

DOI: $10.21883 /$ OS.2019.01.47047.256-18

\section{Введение}

Жидкокристаллические (ЖК) устройства с модальным принципом управления [1,2] представляют интерес в качестве относительно недорогих, технологически простых адаптивных устройств, позволяющих формировать световые поля сложной структуры и динамически управлять их свойствами. Особенностью этих устройств является наличие в их конструкции дополнительного однородного высокоомного прозрачного слоя - управляющего электрода, который наносится на низкоомное покрытие с отверстием. В них используются всего несколько электродов и низкие управляющие напряжения.

Исследования последнего десятилетия привели к созданию новых устройств модального типа, таких как двумерные матрицы управляемых микроаксиконов, параметрами которых можно управлять за счет прикладываемых напряжений [3-5], ЖК спиральные фазовые пластинки для реализации оптических вихрей с высокой энергетической эффективностью $[6,7]$ и других.

Модальный принцип управления реализован и в предложенном нами в работах [8-10] 4-канальном (по числу управляющих контактов) ЖК модуляторе. Такой модулятор позволяет реализовать фокусировку излучения в точку и отрезок (отсюда его альтернативное название ЖК фокусатор), а также формировать световые поля с распределением интенсивности в заданной плоскости в виде колец, эллипсов и $C$-образные световые поля. Управление световыми полями (их размерами, формой, например от кольца к эллипсу и наоборот) и их перемещение по апертуре устройства реализуется за счет изменения прикладываемых напряжений.

ЖК фокусатор характеризуется достаточной лучевой прочностью (эксперименты проводились при плотностях мощности излучения, падающего на фокусатор,

* XIII International Conference on Hole Burning, Single Molecule, and Related Spectroscopies: Science and Applications (HBSM-2018), August 6-12, 2018, Suzdal-Moscow, Russia. до $\left.30 \mathrm{~W} / \mathrm{cm}^{2}\right)$, простотой и компактностью устройства и системы управления и, как следствие, более низкой стоимостью системы по сравнению с коммерческими многоэлементными ПМС. При этом в работах $[11,12]$ было показано, что возможно формирование световых полей с более сложным распределением интенсивности в поперечной плоскости, например в виде границ квадратов, ромбов, параллелограммов и восьмиугольников. Вид распределения определяется выбором режима работы устройства и зависит от величины модального параметра и положения плоскости наблюдения.

В работе [13] продемонстрирована возможность создания с помощью 4-канального ЖК модулятора аксиальносимметричных оптических вихрей (световых колец с орбитальным угловым моментом). Для этого необходимо несколько изменить геометрию контактов электродов ЖК модулятора таким образом, чтобы, подавая специально выбранные значения потенциалов, обеспечить скачок в фазовом профиле пропускания ЖК модулятора.

В настоящей работе мы анализируем возможности ЖК фокусатора по формированию других типов вихревых световых полей, в частности световых полей с распределением интенсивности в виде двух максимумов, вращающихся при распространении - так называемых двухлепестковых световых полей. Такие поля перспективны для ряда прикладных задач и активно исследуются в последнее время [14-20]. Например, с их помощью можно повысить разрешение флуоресцентной микроскопии, они могут быть востребованы в задачах оптической манипуляции. В упомянутых выше работах предлагаются различные методы формирования двухлепестковых пучков с вращением, а для их экспериментальной реализации используются многопиксельные пространственные модуляторы света. В настоящей работе предлагается схема реализации подобных полей с помощью модулятора модального типа и анализируется эффективность их формирования. 


\section{Принцип действия ЖК модулятора для формирования двухлепестковых световых полей}

ЖК фокусатор представляет собой устройство (рис. 1), которое состоит из скрещенных стеклянных подложек, на которые наносят прозрачные высокоомные покрытия (поверхностное сопротивление порядка $100 \mathrm{kOhm} /$ square) и низкоомные непрозрачные полосковые контакты. Подложки располагают так, чтобы их контактные электроды были перпендикулярны друг другу. Между подложками помещают слой нематического ЖК, толщину которого задают прокладками, а первоначальную планарную ориентацию - нанесенными на подложки ориентирующими покрытиями. Управляя амплитудами и фазами потенциалов, прикладываемых к контактам устройства, можно изменять распределение электрического напряжения по апертуре. Под действием напряжения в ЖК слое происходит переориентация молекул ( $S$-эффект). Это приводит к изменению пространственного распределения фазовой задержки, вносимой ЖК слоем в проходящую световую волну.

Для того чтобы с помощью такого модулятора можно было формировать вращающиеся двухлепестковые поля, нужно на одной из подложек (например, нижней) проводящее покрытие и непрозрачные полосковые электроды разделить по середине узкой (около $10 \mu \mathrm{m}$ шириной) непроводящей прозрачной полосой. В результате этого мы получим устройство с шестью контактами.

Для формирования двухлепесткового поля необходимо работать в режиме управления со стационарным сдвигом фаз потенциалов. Для четырехканального модулятора этот режим реализуется при разности фаз на каждой из подложек $\pi$ и разности фаз потенциалов на разных подложках $\pi / 2[10,12]$. Для частного случая квадратной апертуры при соблюдении этих условий и равенстве амплитуд всех потенциалов эквипотенциальные линии напряжения имеют вид концентрических окружностей с центром в точке с координатами $(0,0)$. Из-за порогового характера вольт-фазной характеристики ЖК профиль фазовой задержки будет иметь вид усеченного конуса. При прохождении плоской однородной световой волны через оптический транспарант с таким фазовым пропусканием в области дифракции Френеля на небольшом расстоянии от ЖК фокусатора будет формироваться световое кольцо, размеры которого уменьшаются с ростом расстояния от фокусатора, и в некоторой плоскости распределение интенсивности сфокусируется в точечное пятно.

Если подать на левый и правый контакты одинаковые потенциалы (удовлетворяющие режиму управления со стационарным сдвигом фаз потенциалов), то наличие узкой непроводящей прозрачной полосы не окажет существенного влияния, и рассматриваемое устройство будет работать как 4-канальный ЖК фокусатор. Для реализации с помощью такого модулятора двухлепестко-

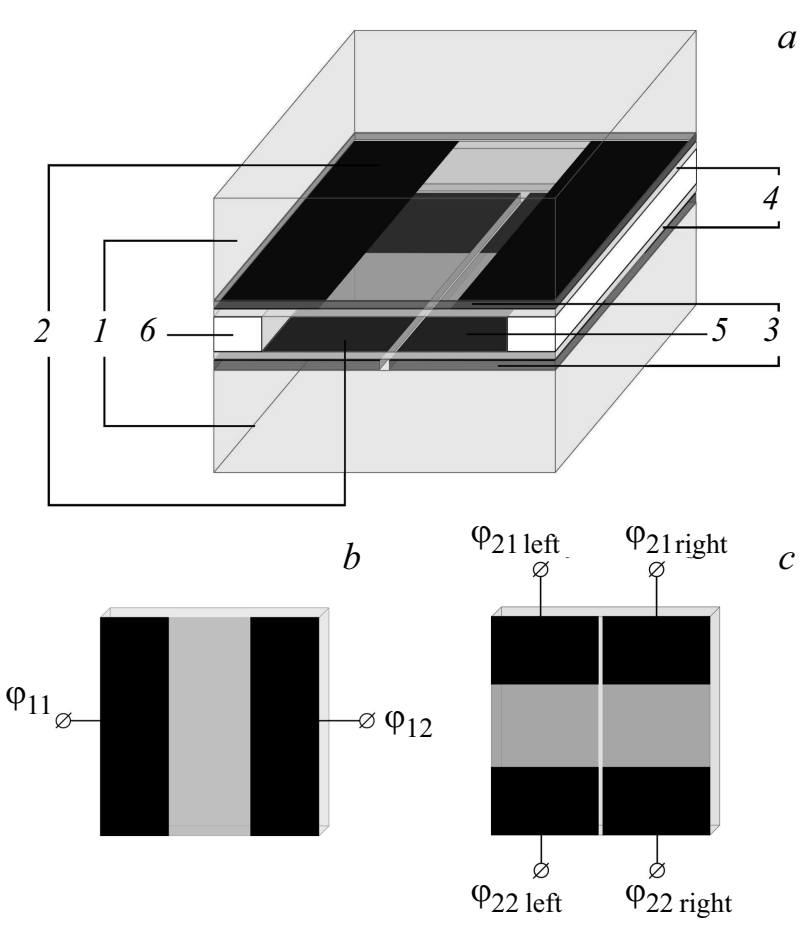

Рис. 1. Схема ЖК фокусатора для формирования вращающихся двухлепестковых световых полей $(a): 1-$ стеклянные подложки, 2 - низкоомные контактные электроды, 3 высокоомный проводящий слой, $4-$ ориентирующее покрытие, 5 - слой ЖК, $6-$ прокладки; геометрия контактов верхней $(b)$ и нижней $(c)$ подложек соответственно: $\varphi_{11}, \varphi_{12}$, $\varphi_{21 \text { left }}, \varphi_{22 \text { left }}, \varphi_{2 \text { 1right }} ; \varphi_{22 \text { right }}-$ потенциалы, подаваемые на соответствующие контакты.

вого светового поля нужно сформировать распределение напряжения с эквипотенциальными линиями в виде концентрических колец с небольшим сдвигом их центров относительно друг друга. Для этого на контакты необходимо подать потенциалы в соответствии с формулой

$$
\begin{gathered}
\varphi_{11}=A_{11} \exp (i 0)=A_{11}, \\
\varphi_{12}=A_{11} \exp (i \pi)=-A_{11}, \\
\varphi_{21 \text { left }}=\left(A_{11}+\Delta\right) \exp \left(i \frac{3 \pi}{2}\right), \\
\varphi_{21 \text { right }}=\left(A_{11}-\Delta\right) \exp \left(i \frac{\pi}{2}\right), \\
\varphi_{22 \text { left }}=\left(A_{11}-\Delta\right) \exp \left(i \frac{\pi}{2}\right), \\
\varphi_{22 \text { right }}=\left(A_{11}+\Delta\right) \exp \left(i \frac{3 \pi}{2}\right),
\end{gathered}
$$

где $\varphi_{11}, \varphi_{12}, \varphi_{21 \text { left }}, \varphi_{22 \text { left }}, \varphi_{21 \text { right }} ; \varphi_{22 \text { right }}$ - потенциалы, подаваемые на контакты ЖК фокусатора в соответствии с рис. $1, b$ и $1, c ; A_{11}, A_{11}+\Delta, A_{11}-\Delta-$ амплитуды потенциалов. Согласно (1), должен выполняться режим управления со стационарным сдвигом фаз потенциалов, 
но значения амплитуд потенциалов на соседних контактах, разделенных непроводящей прозрачной полосой, должны отличаться.

Заданные значения приводят к смещению центров формируемых распределений в противоположные стороны, что позволяет создавать конический профиль действующего значения напряжения и профиль фазовой задержки в виде усеченного конуса (аксикона), половинки которого можно сдвигать в противоположные стороны поперек апертуры. Таким образом, на некотором расстоянии от модулятора световое поле будет иметь распределение интенсивности в поперечной плоскости в виде точечных пятен, расположенных на некотором расстоянии друг от друга. То есть будет формироваться двухлепестковое световое поле. Было выполнено численное моделирование работы устройства для определенных его характеристик и различных значений потенциалов. Результаты численного моделирования приводятся ниже.

\section{Результаты численного моделирования}

При моделировании работы ЖК устройства апертура полагалась квадратной с длиной стороны $2 \mathrm{~mm}$; для расчета фазовой задержки использовалась экспериментальная вольт-фазная характеристика ЖКВL037 [13], нормированная на толщину слоя $\left(l_{L C}\right)$. Моделирование выполнялось для разных значений потенциалов, удовлетворяющих условию (1). Примеры значений потенциалов, подаваемых на контакты, приведены в табл. 1. Рассматривались случаи, когда апертура устройства была ограничена круглой диафрагмой диаметром $D_{\text {ап }}$ (строки 3, 4 в табл. 1) и не ограничивалась диафрагмой (строки 1,2). Диаметр апертуры полагался равным $2 \mathrm{~mm}$. Толщина слоя задавалось равной 20 (строки $1-3)$ и $5 \mu \mathrm{m}$ (строка 4). Также в таблице приведены расстояния от фокусатора до плоскости наблюдения $(z)$.

На рис. 2 приведены соответствующие распределения напряжения по апертуре $(a)$, фазовой задержки $(b)$, поляризационная интерферограмма $(c)$, а также примеры распределения интенсивности $(d)$ и фазы $(e)$ светового поля, формируемого фокусатором в плоскости наблюдения для значений потенциалов, представленных в строке № 1 табл. 1 .

Видно, что из-за напряжения ниже порогового для ЖК в центральной части получается усеченный профиль фазовой задержки. При прохождении световой волны через такой фазовый транспарант формируется световое поле с распределением интенсивности в поперечной плоскости в виде двух максимумов (рис. $2, d$ ) и скачками в распределении фазы (рис. 2,e), т.е. формируется вращающееся двухлепестковое вихревое поле.

Примеры рассчитанных световых полей в поперечных плоскостях, расположенных на различных расстояниях от фокусатора, представлены на рис. 3. Расчеты проводились в предположении, что на транспарант падает плоская однородная световая волна. На представленных изображениях видно, что в распределении интенсивности присутствуют два максимума, которые с изменением расстояния изменяют свое положение, поворачиваясь вокруг центра картины.

Была промоделирована другая ситуация, когда плоскость наблюдения неподвижна, а изменяется кривизна волнового фронта падающей световой волны, которую мы характеризовали величиной $1 / r$, где $r$ - радиус кривизны волнового фронта падающей световой волны. Эта ситуация интересна с точки зрения возможного применения двухлепестковых полей, формируемых ЖК модулятором, для повышения разрешающей способности микроскопов. В случае, когда источник излучения находится в фокусе линзы, на ЖК фокусатор падает плоская волна $\left(1 / r=0 \mathrm{~m}^{-1}\right)$, при смещении источника из фокуса формируется сходящийся либо расходящийся волновой фронт, кривизна которого $(1 / r)$ меняется в зависимости от величины смещения. Результаты моделирования показали, что наблюдается картина, аналогичная рис. 3 - максимумы интенсивности поворачиваются вокруг своей оси в зависимости от величины $1 / r$.

Пример графиков зависимости угла поворота пучка $\theta$ от $1 / r$ для параметров ЖК фокусатора, приведенных в табл. 1, представлен на рис. 4. Под углом поворота пучка мы понимали угол между линией, проходящей через центры масс пятен в распределении интенсивности, и горизонтальной линией (вкладка на рис. 4). На левой вкладке представлено типичное распределение интенсивности светового поля, формируемого фокусатором в плоскости наблюдения. На правой вкладке изображение отфильтровано по уровню падения интенсивности 0.5 . Именно по этому обработанному изображению определялся угол поворота.

Полученные графики были проанализированы с точки зрения оценки возможности использования модифицированного ЖК фокусатора для увеличения продольного разрешения флуоресцентных микроскопов, а именно для оценки продольного смещения объектов друг относительно друга в предметной области по изменению положения максимумов интенсивности в плоскости изображения. Результаты представлены в табл. 2. Интерес представляют диапазон углов (Range_ $\left.\theta_{\text {full }}\right)$, который может быть реализован с помощью ЖК фокусатора, или полный угол поворота $\left(\theta_{\text {full }}\right)$. Заметим, что при определении этих величин мы не принимали во внимание разрывы в графиках зависимости $\theta(1 / r)$. Наличие этих разрывов связано с тем, что при определенных значениях кривизны падающего светового поля в картине дифракции в плоскости наблюдения наряду с основными максимумами интенсивности наблюдаются дополнительные максимумы, либо происходит размытие основных максимумов, что затрудняет определение угла поворота. В какой-то степени наличие этих разрывов связано со способом обработки изображения, например, если фильтровать изображение по уровню падения интенсивности 0.75 , то разрывы в зависимости не наблюдаются. 
Таблица 1. Параметры ЖК фокусатора

\begin{tabular}{|c|c|c|c|c|c|c|c|c|c|c|c|c|c|}
\hline № & $A_{11}, \mathrm{~V}$ & $\alpha_{11}$ & $A_{12}, \mathrm{~V}$ & $\alpha_{12}$ & $A_{12 \text { left }}, \mathrm{V}$ & $\alpha_{12 \mathrm{left}}=a_{22 \text { right }}$ & $A_{21 \text { right }}, \mathrm{V}$ & $\alpha_{21 \text { right }}=\alpha_{22 \text { left }}$ & $A_{22 \text { left }}, \mathrm{V}$ & $A_{22 \text { right }}, \mathrm{V}$ & $D_{a n}, \mathrm{~mm}$ & $z, \mathrm{~cm}$ & $l_{L C}, \mu \mathrm{m}$ \\
\hline 1 & 3.0 & 0 & 3.0 & $\pi$ & 3.2 & $3 \pi / 2$ & 2.8 & $\pi / 2$ & 2.8 & 3.2 & - & 24 & 20 \\
\hline 2 & 5.0 & 0 & 5.0 & $\pi$ & 5.2 & $3 \pi / 2$ & 4.8 & $\pi / 2$ & 4.8 & 5.2 & - & 12 & 20 \\
\hline 3 & 3.0 & 0 & 3.0 & $\pi$ & 3.2 & $3 \pi / 2$ & 2.8 & $\pi / 2$ & 2.8 & 3.2 & 2 & 24 & 20 \\
\hline 4 & 3.0 & 0 & 3.0 & $\pi$ & 3.5 & $3 \pi / 2$ & 2.5 & $3 \pi / 2$ & 2.5 & 3.5 & 2 & 46 & 5 \\
\hline
\end{tabular}

Примечание. $A_{11}, A_{12}$ - амплитуды потенциалов, $\alpha_{11}, \alpha_{12}$ - фазы потенциалов, подаваемых на контакты подложки со сплошным электродом; $A_{21 \text { left }}, A_{22 \mathrm{left}}$ - амплитуды потенциалов, $\alpha_{21 \mathrm{left}}, \alpha_{22 \mathrm{left}}-$ фазы потенциалов, подаваемых на контакты левой половинки подложки с узкой непроводящей полосой; $A_{21 \text { right }} ; A_{22 \text { right }}$ - амплитуды потенциалов, $\alpha_{21 \text { right }} ; \alpha_{22 \text { right }}$ ф фазы потенциалов, подаваемых на контакты правой половинки подложки с узкой непроводящей полосой.

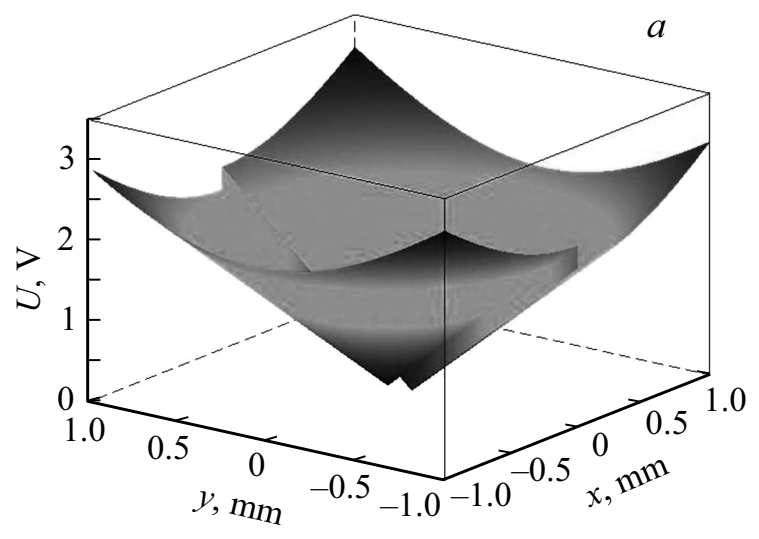

$c$
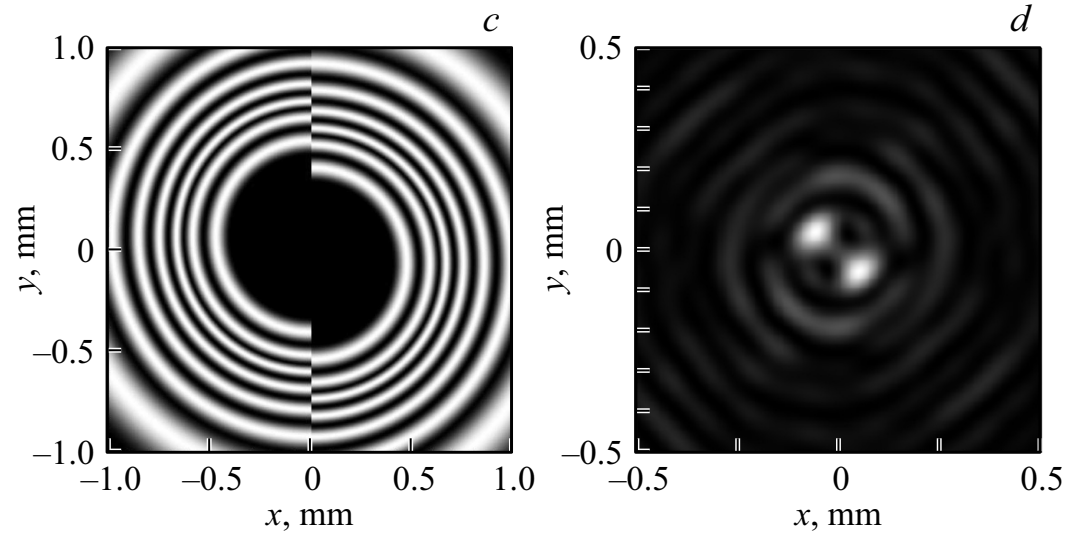

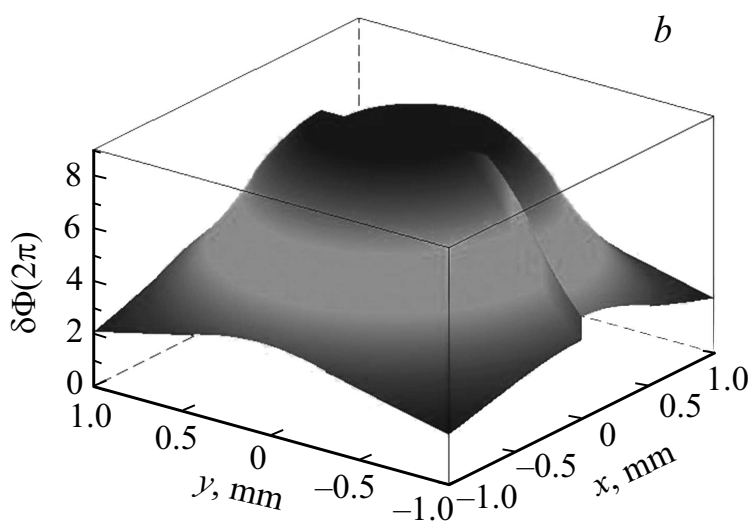

$d$

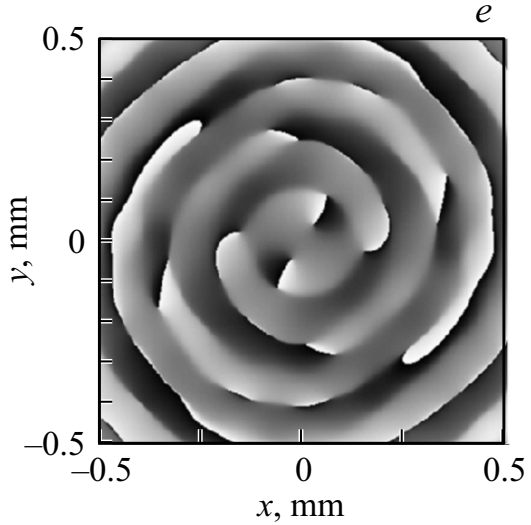

Рис. 2. Распределения напряжения по апертуре $(a)$, фазовая задержка $(b)$, поляризационная интерферограмма $(c)$, распределения интенсивности $(d)$ и фазы $(e)$ поля, формируемые фокусатором на расстоянии $24 \mathrm{~cm}$. Значения амплитуд и фаз потенциалов, подаваемых на контакты ЖК фокусатора, приведены в строке № 1 табл. 1.
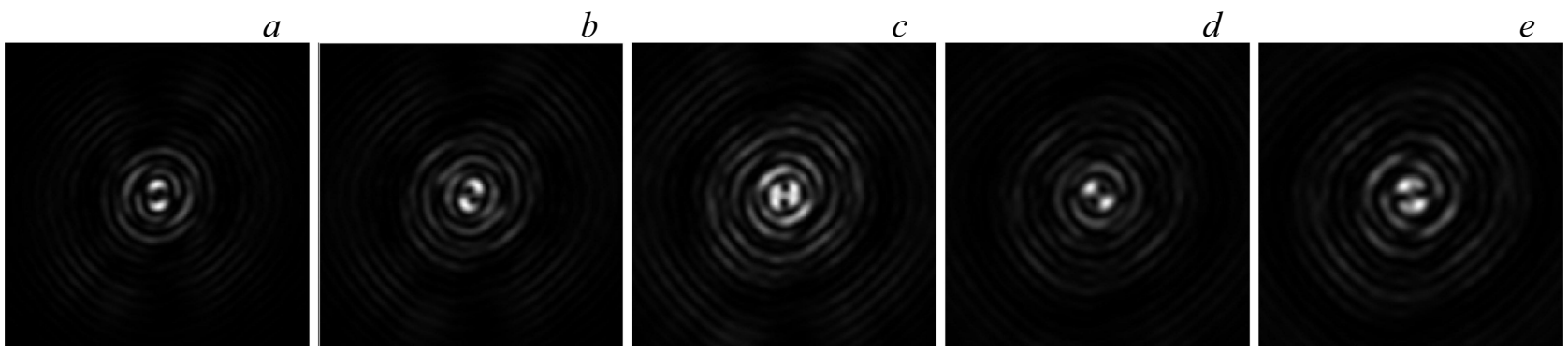

Рис. 3. Распределения интенсивности светового поля, формируемого ЖК фокусатором в плоскости наблюдения в зависимости от ее положения. Расстояние от ЖК фокусатора до плоскости наблюдения $20(a), 22(b), 24(c), 26(d)$ и $28 \mathrm{~cm}(e)$. 
Таблица 2.

\begin{tabular}{c|c|c|c|c|c|c|c|c}
\hline № & Range_ $\theta_{\text {full }}$ & $\theta_{\text {full }}$ & $V_{\theta},{ }^{\circ} / \mathrm{m}^{-1}$ & Range_ $\theta_{\text {line }}$ & $1 / r, \mathrm{~m}^{-1}$ & $R^{2}$ & $P_{\max }, \%$ & $P_{\min }, \%$ \\
\hline 1 & $104^{\circ}-\left(-215^{\circ}\right)$ & $319^{\circ}$ & 289 & $-58^{\circ}-\left(-116^{\circ}\right)$ & $0.6-0.8$ & 0.9999 & 7 & 5 \\
2 & $142^{\circ}-(-45)^{\circ}$ & $187^{\circ}$ & 50.5 & $142^{\circ}-\left(-45^{\circ}\right)$ & $-2.5-1.6$ & 0.9935 & 4 & 2 \\
3 & $147^{\circ}-(-45)^{\circ}$ & $192^{\circ}$ & 107 & $-13^{\circ}-\left(-151^{\circ}\right)$ & $0.1-1.4$ & 0.994 & 4 & 6 \\
4 & $138^{\circ}-30^{p}$ & $108^{\circ}$ & 90 & $110^{\circ}-49^{\circ}$ & $-0.2-0.5$ & 0.996 & 50 & 37
\end{tabular}

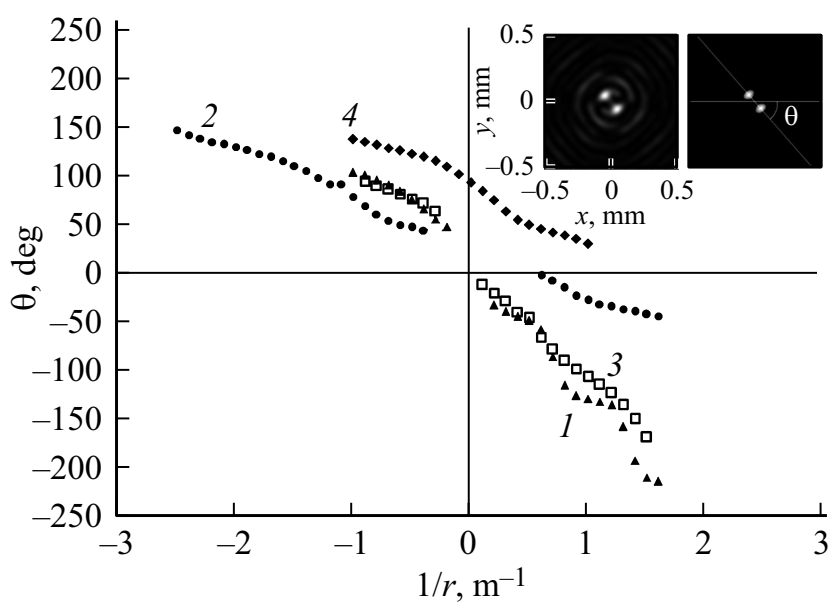

Рис. 4. График зависимости угла поворота $\theta$ сформированного пучка от кривизны волнового фронта падающего пучка. Номера кривых соответствуют номерам строк в табл. 1 и получены для соответсвующих параметров ЖК фокусатора.

Также в таблице приведена так называемая максимальная скорость $\left(V_{\theta}\right)$ поворота двухлепесткого пучка в зависимости от кривизны волнового фронта падающего пучка. Величина определялась как тангенс угла наклона линейного участка графика зависимости. $R^{2}-$ коэффициент достоверности аппроксимации. C практической точки зрения наибольший интерес представляет диапазон углов, соответствующий линейному участку с максимальной скоростью поворота (Range_ $\left.\theta_{\text {line }}\right)$. Кроме того, оценивались энергетические эффективности полученных пучков: в таблице приведены максимальные $\left(P_{\max }\right)$ и минимальные $\left(P_{\min }\right)$ значения доли мощности в пятнах по уровню падения интенсивности 0.5 для рассматриваемого линейного участка. Данные приведены для параметров фокусатора, представленных в табл. 1 (номера строк в таблицах соответствуют друг другу).

Анализ зависимостей показывает, что ограничение апертуры ЖК фокусатора круглой диафрагмой (см. строки 1 и 3) приводит к тому, что зависимости становятся более плавными, линейные участки увеличиваются, хотя зависимость угла поворота сформированного пучка от радиуса кривизны падающего пучка становится менее выраженной (скорость угла поворота в зависимости от кривизны волнового фронта становится меньше). Наличие апертуры не влияет на энергетическую эффективность сформированных полей (доля мощности в максимумах интенсивности для параметров ЖК фокусатора, представленных в строках 1-3 табл. 1, является довольно низкой). Однако за счет выбора параметров фокусатора - величин приложенных напряжений, а также толщины ЖК слоя - можно повысить энергетическую эффективность до 50\% (см. строку 4). Эти величины являются сопоставимыми с данными, приведенными в [21], где двухлепестковые вихревые пучки формировались с помощью фазового пространственного ЖК модулятора HOLOEYE HEO-1080, а соответствующие фазовые маски рассчитывались с использованием оптики спиральных пучков. Измеренные энергетические эффективности в зависимости от параметров пучка составили от 27 до 56\%. В нашем случае повышение доли мощности излучения в пятнах привело к уменьшению полного угла поворота пучка (он составил лишь $108^{\circ}$ ) и скорости поворота $\left(90 \% \mathrm{~m}^{-1}\right)$.

Случай, представленный в строке 2, демонстрирует, что выбором напряжений, прикладываемых к контактам ЖК модулятора, можно достичь того, что зависимость становится непрерывной, т.е. для всех значений кривизны волнового фронта в распределении интенсивности наблюдаются два довольно четких максимума. Кроме того, скорость угла поворота становится постоянной, т. е. зависимость угла поворота от величины, обратной радиусу кривизны падающего на фокусатор волнового фронта, аппроксимируется линейной функцией с хорошей точностью во всем диапазоне значений $1 / r$. Для рассматриваемого случая полный угол поворота при этом составил около $190^{\circ}$, а скорость угла поворота $50.5^{\circ} / \mathrm{m}^{-1}$.

\section{Заключение}

В работе представлена схема модального ЖК устройства для формирования двухлепестковых световых полей с вращением. В основе устройства лежит предложенный авторами ранее ЖК фокусатор. Для реализации световых полей в виде двух световых максимумов необходимо проводящее покрытие и непрозрачные полосковые электроды на одной из подложек фокусатора разделить по середине узкой непроводящей прозрачной полосой и подать на контакты устройства определенным образом выбранные напряжения.

Изменение положения максимумов при изменении радиуса кривизны падающей волны указывает на возможность использования предлагаемого модифициро- 
ванного ЖК фокусатора в системе микроскопа для оценки продольного смещения объектов друг относительно друга в предметной области по изменению положения максимумов интенсивности в плоскости изображения. Однако для этого необходимо повысить энергетическую эффективность формируемых пучков, а также сформировать пучки с большей скоростью угла поворота. Результаты численного моделирования показали, что эти величины изменяются за счет изменения параметров ЖК устройства (толщины ЖК слоя и прикладываемых к контактам напряжений). Однако возможность реализации значений, представляющих реальный практический интерес, требует дальнейшего изучения.

Работа выполнена при поддержке РФФИ (гранты № 16-02-01055, № 16-29-11809 и № 16-42-630773).

\section{Список литературы}

[1] Абрамочкин Е.Г., Васильев А.А., Вашурин П.В., Жмурова Л.И., Игнатов В.А., Наумов А.Ф. // Препринт ФИАН. 1988. T. 194. $18 \mathrm{c}$.

[2] Naumov A.F., Loktev M.Y., Guralnik I.R., Vdovin G. // Opt. Lett. 1998. V. 23. P. 992. doi 10.1364/OL.23.000992

[3] Algorri J.F., Urruchi V., Bennis N., Sánchez-Pena J.M., Otón J.M. // Opt. Express. 2015. V. 23. P. 13899. doi 10.1364/OE.23.013899

[4] Algorri J.F., Love G.D., Urruchi V. // Opt. Express. 2013. V. 21. P. 24809. doi 10.1364/OE.21.024809

[5] Algorri J.F., Urruchi V., Bennis N., Morawiak P., SánchezPena J.M., Otón J.M. // Opt. Express. 2017. V. 25. P. 605. doi 10.1364/OE.25.000605

[6] Algorri J.F., Urruchi V., Garcia-Cámara B., SánchezPena J.M. // Electron Device Letters. IEEE. 2014. V. 35. P. 856.

[7] Albero J., Garcia-Martinez P., Bennis N., Oton E., Cerrolaza B., Moreno I., Davis J.A. // J. Lightwave Technol. 2012. V. 30. P. 3055.

[8] Котова С.П., Патлань В.В., Самагин С.А. // Квант. электрон. 2011. T. 41. № 1. C. 58; Kotova S.P., Patlan V.V., Samagin S.A. // Quant.Electron. 2011. V. 41. N 1. P. 58.

[9] Котова С.П., Патлань В.В., Самагин С.А. // Квант. электрон. 2011. T. 41. № 1. C. 65; Kotova S.P., Patlan V.V., Samagin S.A. // Quant. Electron. 2011. V. 41. N 1. P. 65.

[10] Kotova S.P., Mayorova A.M., Samagin S.A. // J. Optics. 2015. V. 17. N 5. P. 055602. doi 10.1088/2040-8978/17/5/055602

[11] Котова С.П., Майорова А.М., Самагин С.А. // Оптический журнал. 2017. T. 84. № 5. C. 46; Kotova S.P., Mayorova A.M., Samagin S.A. // J. Optical Technology. 2017. V. 84. N 5. P. 323. doi 10.1364/JOT.84.000323

[12] Kotova S.P., Mayorova A.M., Samagin S.A. // Proc. SPIE. Asia-Pacific Conference on Fundamental Problems of Optoand Microelectronics (APCOM 2016). Khabarovsk, Russia, 2016. V. 10176. P. 1017626. doi 10.1117/12.2268180

[13] Kotova S.P., Mayorova A.M., Samagin S.A. // J. Optics. 2018. V. 20. N. P. 055604. doi 10.1088/2040-8986/aab8bb

[14] Pavani S.R.P., Piestun R. // Opt. Express. 2008. V. 16. N 5. P. 3484. doi 10.1364/OE.16.003484

[15] Baránek M., Bouchal P., Šiler M., Bouchal Z. // Opt. Express. 2015. V. 23. N 12. P. 15316. doi 10.1364/OE.23.015316
[16] Roider C., Jesacher A., Bernet S., Ritsch-Marte M. // Opt. Express. 2014. V. 22. N 4. P. 4029. doi 10.1364/OE.22.004029

[17] Naumov A.V., Eremchev I.Y., Gorshelev A.A. // Europ. Phys. J. D. 2014. V. 68. N 11. P. 348.

[18] Воронцов Е.Н., Котова С.П., Лосевский Н.Н., Прокопова Д.В., Самагин С.A. // Краткие сообщения по физике. 2018. № 3. С. 9; Vorontsov E.N., Kotova S.P., Losevsky N.N., Prokopova D.V., Samagin S.A. // Bulletin of the Lebedev Physics Institute. 2018. V. 45. N 3. P. 71. doi 10.3103/S1068335618030028

[19] Bouchal P., Štrbková L., Dostál Z., Bouchal Z. // Opt. Express. 2017. V. 25. N 18. P. 21428. doi 10.1364/OE.25.021428

[20] Wang Z., Cai Y., Liang Y., Zhou X., Yan S., Dan D., Bianco P.R., Lei M., Yao B. // Biomed. Opt. Express. 2017. V. 8. N 12. P. 5493. doi 10.1364/BOE.8.005493

[21] Воронщов Е.Н., Лосевский Н.Н., Прокопова Д.В., Разуева Е.В., Самагин С.А. // Компьютерная оптика. 2016. Т. 40. № 2. C. 158. doi 10.18287/2412-6179-2016-40-2-158-163 